\title{
INVESTIGAÇÃO DOS NÍVEIS DE EFICIÊNCIA NA ALOCAÇÃO DOS RECURSOS PÚBLICOS APÓS O PACTO PELA SAÚDE ${ }^{1}$
}

\author{
RESEARCH LEVELS OF EFFICIENCY IN THE ALLOCATION OF PUBLIC FUNDS \\ AFTER THE HEALTH PACT
}

\section{NIVELES DE INVESTIGACIÓN DE LA EFICIENCIA EN LA ASIGNACIÓN DE RECURSOS DE SALUD PÚBLICA DESPUÉS EL PACTO}

\author{
Kerla Fabiana Dias Cabral \\ Universidade Federal de Viçosa \\ kerlacabral@hotmail.com
}

\author{
Marco Aurélio Marques Ferreira \\ Universidade Federal de Viçosa \\ marcoaurelio@ufv.br
}

\begin{abstract}
Resumo: O presente estudo toma como referência o contexto político derivado das alterações experimentadas pelo setor de saúde do Brasil a partir da Constituição Federal de 1988 em especial, decorrentes do processo de descentralização, que realçaram a necessidade de se utilizar osrecursos municipais mais eficientemente, nesse contexto é criado em 2006, como estratégia de racionalização das ações e serviços em saúde o Pacto pela Saúde.Nessa vertente o presente estudo buscou analisar os níveis de eficiência na utilização dos recursos de saúde após o Pacto, no qual estados e municípios passaram a receber recursos federais por meio de cinco blocos de financiamento. O método utilizado foi o Data Envelopment Analisys (DEA),objetivando comparar e quantificar a eficiência no uso de recursos no setor de saúde de 30 municípios mineiros que aderiram ao Pacto. Os resultados demonstraram que o desempenho na alocação dos recursos nos municípios analisados pode ser considerado bom, pois a maioria dos municípios concentrou-se em escores superiores à média, concluindo-se que o modelo de gestão proposto pelo Pacto pela Saúde influencia positivamente a alocação dos recursos nos setores analisados.
\end{abstract}

Palavras-Chave: Pacto pela saúde. Política Pública. Alocação de recursos.

\begin{abstract}
The present study takes as a benchmark derived from the political changes experienced by Brazil's health sector from the Constitution of 1988 in particular, under the decentralization process, which highlighted the need to use resources more efficiently municipal, in this context is created in 2006 as a strategy for rationalization of activities and services in the health Pact for Health in this strand the present study investigates the levels of efficiency in the use of health resources after the Covenant, in which states and municipalities now receive federal funds through five blocks of funding. The method was used Data Envelopment Analysis (DEA), to compare and quantify the efficiency in the use of resources in the health sector in 30 municipalities miners who joined the Covenant. The results showed that performance in the allocation of resources in the municipalities analyzed can be considered good, since most municipalities focused on above-average scores, concluding that the management model proposed by Health Pact positively influences the allocation of resources in the sectors analyzed.
\end{abstract}

Keywords: Pact for health. PublicPolicy. Allocating resources.

Resumen: El presente estudio toma como referencia el contexto político derivado de los cambios experimentados por el sector de la salud en Brasil a partir de la Constitución Federal de 1988 , en particular, que surjan del proceso de descentralización, que hizo hincapié en la necesidad de utilizar de manera más eficiente osrecursos municipales, esta contexto se creó en 2006 como una estrategia para racionalizar las acciones y servicios de salud en el presente Pacto por Saúde.Nessa este estudio tuvo como objetivo analizar el nivel de eficiencia en el uso de los recursos de salud después de que el Pacto, en el que los estados y municipios

\footnotetext{
${ }^{1}$ Submetido em 03 de abril de 2013. Aceito em 07 de outubro de 2013. O artigo foi avaliado segundo o processo de duplo anonimato além de ser avaliado pelo editor. Editores responsáveis: Márcio Augusto Gonçalves e Lucas Maia dos Santos. Reprodução parcial ou total e trabalhos derivativos permitidos com a citação apropriada da fonte.
} 
han recibido recursos federales a través de cinco bloques de financiación. El método utilizado fue el Análisis Envolvente de Fecha (DEA ), con el objetivo de comparar y cuantificar la eficiencia en el uso de los recursos en el sector salud 30 mineros municipios que se han adherido al Pacto. Los resultados mostraron que el rendimiento en la asignación de recursos en los municipios analizados puede considerarse buena, ya que la mayoría de los municipios se concentraron en las puntuaciones superiores a la media y concluyó que el modelo de gestión propuesto por el Pacto de Salud influye positivamente en la asignación de recursos de los sectores analizados . Palabras Clave : Pacto por la salud. Políticas Públicas. Asignación de recursos .

\section{INTRODUÇÃO}

O Sistema Único de Saúde (SUS) compõe um contemporâneo modelo de organização dos serviços de saúde que possui como uma de suas particularidades a valorização do nível municipal. Entretanto, mesmo com seu alcance social, a sua plena implantação ainda não foi possível, fato decorrente das sérias dificuldades de financiamento e eficiência administrativa de sua operação, sendo recorrente a discussão sobre a ampliação do financiamento da saúde e a melhor utilização dos recursos (COUTTOLENC; ZUCCHI, 1998).

Ao se analisar a situação do financiamento do setor de saúde encontram-se as questões da insuficiência de recursos e baixo grau de eficiência e eficácia em sua aplicação assim como baixa prioridade concedida pelo governo federal. (MARQUES,1999)

Inúmeras e constantes são as tentativas de aumento de mecanismos que levem ao controle dos recursos, à otimização dos serviços de saúde e ao acesso dos usuários a todos os níveis de atenção do sistema, no entanto, a questão assume uma forte complexidade ao considerarem-se as enormes desigualdades geográficas, culturais e socioeconômicas existentes no país (FADEL et al., 2009).

A descentralização no setor de saúde, iniciada em 1988, trouxe a chance de ajustar essas distorções, mas para que isso se efetive, é necessário que o gestor municipal do sistema de saúde incorpore as melhores modalidades e práticas de gestão financeira, garantindo a capacidade técnica e operativa para gerenciar recursos financeiros, além de explorar modalidades alternativas para esse gerenciamento (COUTTOLENC; ZUCCHI, 1998).

$\mathrm{Na}$ busca por uma modalidade mais prática e eficiente de gestão de recursos que auxilie os gestores a alcançar um nível satisfatório na utilização dos recursos financeiros do setor de saúde, em 2006, foi criado o Pacto pela Saúde, dessa forma, os municípios passaram a receber seus recursos por meio de seis blocos de financiamento, substituindo as mais de cem formas de repasse existentes anteriores (FADEL et al., 2009).

Nesse contexto, torna-se relevante abordar a seguinte questão: Após a implementação do pacto de saúde quais os níveis de eficiência satisfatórios no setor de saúde?

\section{REVISÃO DE LITERATURA}

\subsection{POLÍTICA PÚBLICA E O SETOR DE SAÚDE}

A política pública é considerada um fluxo de decisões públicas, dirigido a sustentar o equilíbrio social ou a inserir desequilíbrios com o objetivo de alterar essa realidade. São deliberações dependentes do próprio fluxo e pelas reações e alterações que elas geram na organização social, assim como pelos valores, ideias e visões daqueles que adotam (SARAIVA, 2006).

As políticas públicas, de maneira geral, repercutem na economia e nas sociedades, dessa forma, toda teoria da política pública necessita também elucidar as inter-relações entre economia, sociedade, política e Estado. (SOUZA, 2006)

No que tange à responsabilidade pública no campo da saúde, um aspecto que deve ser lembrado diz respeito à definição das prioridades nos investimentos do Estado, abrangendo o 
estudo da destinação, alocação, distribuição e controle dos recursos financeiros conduzidos ao setor. (GARRAFA et al.,1997).

No Brasil, a problemática do financiamento de saúde se destaca, a partir da Constituição de 1988, quando a saúde passa a ser um direito de todos e dever do Estado, assim a garantia de financiamento de saúde passa de 30 milhões para 150 milhões de segurados (CARVALHO, 2008).

Na década de 1980, o país viveu seu período de redemocratização, e junto a ele o movimento sanitário. Nesse contexto, se deu a discussão da $8^{\text {a }}$ Conferência Nacional de Saúde, que resultou na proposta da integração das ações de saúde e indicou a implantação do Sistema Único de Saúde (SUS), instituído pela Constituição Brasileira de 1988, disciplinado pela Lei No 8080 (BARROSO, 2007).

A universalização trazida pela Constituição Federal de 1988 pode ser avaliada como uma das mais inclusivas das políticas sociais no país, entretanto, as dificuldades econômicas e conflitos políticos convividos no processo de institucionalização do SUS vêm atrapalhando sua viabilidade. (MARQUES; MENDES, 2005)

O SUS é organizado de acordo com as seguintes diretrizes: atendimento integral, descentralização e participação popular. A concepção do SUS representou uma melhoria, sobretudo por seus princípios de organização que demonstram garantia de acesso de toda a sociedade aos serviços de saúde e participação dos cidadãos no processo de formulação de políticas de saúde e do cumprimento das mesmas. (FONTOURA; MAYER, 2006)

Nos anos de 1990 foi criada aOSS como objetivo possibilitar uma maior variedade de fontes para financiar o setor e diminuir dessa forma a vulnerabilidade frente aos períodos econômicos recessivos, entretanto não ofereceu solução ao problema. (LUCCHESE, 1996)

Devido aos graves problemas no orçamento da área da saúde, foi criado, em 1994, como medida emergencial, o Imposto Provisório sobre Movimentações Financeiras-IPMF, que apesar de possuir caráter cumulativo era defendido pelo seu baixo custo e elevado desempenho em termos de arrecadação, vigorou pelo período de um ano e, depois, foi recriado, em 1997, como Contribuição Provisória sobre a Movimentação Financeira - CPMF (CARVALHO, 2001).

A CPMF foi criada como medida provisória na busca por aumento do orçamento da saúde no qual todo recurso arrecado deveria ser destinado a ela, todavia com as inúmeras prorrogações, a contribuição perdeu sua finalidade original, observando-se desvios dos recursos para o Ministério da Educação e o da Defesa dentre outros, havendo diminuição progressiva da sua utilização na saúde ao longo dos anos. (SANTI et al., 2008)

De acordo o setor de saúde recebeu, em 2006, somente cerca de 40\% dos R \$ 32.090 bilhões (US\$ 13.645 bilhões) arrecadados pela CPMF e um volume considerável dos fundos restantes foi usado para o pagamento de juros da dívida pública. (PAIM et. al., 2011)

A criação da CPMF, no Brasil, foi explicada, na visão de seus defensores, pela sua abrangência e simplicidade, por incidir sobre a economia informal, entretanto, os princípios gerais das Finanças Públicas e do Direito Tributário a desaprovam pela desigualdade tributária, a cumulatividade e dupla incidência, a ausência de seletividade e a falta de progressividade (ALBUQUERQUE, 2001).

Contrariando a vontade do Governo Federal, em 2007, a CPMF foi extinta pelo Congresso Nacional, o que levou a um rearranjo das fontes de financiamento para a saúde nos anos posteriores. Determinadas medidas foram adotadas para compensar as perdas financeiras, como o aumento das alíquotas do Imposto sobre Operações Financeiras e da CSLL (MEDICI, 2009).

A Constituição definiu que cada esfera de Governo deveria aplicar parte dos recursos de seus orçamentos em saúde, porém não fixou os percentuais e nem o que era gasto em 
saúde, o que abriu brecha para que os recursos fossem desviados para outros fins (CARVALHO, 2008).

Pela falta de um parâmetro legal que obrigasse os Estados, Distrito Federal e municípios a destinarem recursos próprios para a área de saúde, as fontes de financiamento no âmbito da União eram instáveis, e de forma recorrente adotavam medidas emergenciais e provisórias, objetivando a não repetição desses problemas, no ano 2000, foi aprovada a Emenda Constitucional no 29 - EC 29, que alterou artigos da Constituição e acrescentou um ao Ato das Disposições Constitucionais Transitórias, para garantir os recursos mínimos para o financiamento da saúde (CAMPELLI; CALVO, 2007).

A emenda constitucional 29 estabelece que os estados devam investir $12 \%$ da receita de impostos estaduais em serviços e ações de saúde; os municípios, $15 \%$ da receita de impostos municipais; e a União, o montante aplicado no ano anterior corrigido pela variação nominal do produto interno bruto (PIB) (PASCHE et al.,2006).

Com a chegada da EC $n^{\circ} 29$, notou-se uma mudança gradativa na proporção da participação da União no financiamento da saúde no que tange a municípios e estados. Nas décadas de 1980 e 1990, cabia à União mais de 70\% do gasto público do setor de saúde. No ano de 2000, encontrava-se em $60 \%$ e, em 2003 e 2004, esta participação diminuiu para cerca de 50\%. (FRUTUOSO, 2010)

No que diz respeito aos municípios, nota-se que a maior parte está se aproximado do seu limite e capacidade de investimento próprio em saúde, junto ao fato de que o orçamentobase da EC-29, na esfera municipal, tem se elevado menos que seu orçamento geral, o que os está obrigando a investir mais que os percentuais preconizados pela Emenda, o que pode caracterizar também um obstáculo à regionalização (LEVI; SCATENA, 2010).

Para melhor entendimento sobre as tentativas de operacionalização do SUS, a seguir será tratado sobre o Pacto pela Saúde.

\subsection{PACTO PELA SAÚDE}

Oprocesso de descentralização iniciado em 1988 trazido pela Constituição Federal buscava uma maior adequação no atendimento das necessidades das populações locais, e uma melhora na eficiência alocativa dos recursos. PEIXOTO et al., (2012)

Entretanto processo de descentralização apresenta algumas debilidades sendo as principais o enfraquecimento das estruturas principais, dificuldade de coordenação, o aumento dos custos de transação, a fragmentação dos serviços, a ineficiência por perda de economia de escala e de escopo, a insuficiência de recursos gerenciais, o clientelismo local, e a desestruturação de programas verticais de saúde pública (PRUD`HOMME, 1995).

Para orientar intenso processo de descentralização foram criadas as Normas Operacionais Básicas (NOBs) do SUS, que apresentaram algumas fragilidades do processo como dificuldades no estabelecimento de fluxos de referência e contra referência, de categoria e de conexão dos serviços de saúde. (LEVCOVITZ; LIMA; MACHADO, 2001).

Considerando as dificuldades do processo de descentralização, em 2001, foi publicada, no Brasil, uma norma operacional de assistência à saúde, a Noas SUS 2001/01, tendo como objetivo geral promover maior equidade na alocação de recursos e acesso da população a todos os níveis de atenção, dando proeminência à formação de redes integradas e apontando para o reconhecimento da regionalização da atenção à saúde como uma macro estratégia para consolidação dos objetivos (SOUZA, 2001).

A falta de mecanismos para a adesão das novidades trazidas pela Noas SUS 01/01 levam a publicação da Noas SUS 01/02 que oferece alternativas viáveis à superação dos entraves originários de sua implementação, por meio da regulação da assistência agora apresentado de forma mais completa (FADEL et al., 2009). 
Apesar dos esforços das três esferas de gestão na implementação da Noas 01/02, notava-se um descompasso nesse processo, uma vez que, nem todos os estados se encontram no mesmo momento de evolução, surgindo a necessidade de uma nova estratégia de operacionalização do SUS no Brasil. No intuito de suprir esse desacordo evolutivo, em 2006, é editada a portaria no 339 que torna público o Pacto pela Saúde (FADEL et al., 2009).

O Pacto pela Saúde - PS representa mudanças operacionais de forma a efetivar acordos nas três esferas de gestão do SUS, tendo como objetivo inovar processos e instrumentos de gestão, redefinir as responsabilidades coletivas por resultados sanitários, pautando-se pelas necessidades da população além de promover a qualidade social e sanitária à população.(PEREIRA et al., 2009)

O PS traz como inovação o fim da habilitação para estados e municípios. Em seu contexto, visa estabelecer de modo mais preciso, a responsabilização solidária no SUS, no qual todos os gestores são considerados plenos de suas responsabilidades, suprindo os processos de habilitação precedentes pela adesão ao Termo de Compromisso e Gestão (TCG) (GUERREIO; BRANCO, 2011).

Dentre seus objetivos gerais o Pacto pela Saúde visa enumerar, aprimorar e definir responsabilidades sanitárias e de gestão por meio de três componentes, sendo eles o pacto pela vida (PV), o pacto em defesa do SUS (Pdsus) e o pacto de gestão (PG). (FADEL et al., 2009)

O PV prioriza a Saúde do Idoso; Controle do câncer do colo do útero e da mama; Redução da mortalidade infantil e materna; Fortalecimento da capacidade de resposta às doenças emergentes e endemias, com ênfase na dengue, hanseníase, tuberculose, malária e influenza; Promoção da Saúde e Fortalecimento da Atenção Básica. (PEREIRA et.al., 2009)

A segunda dimensão é o pacto em defesa do SUS que passar a existir como um movimento de repolitização e mobilização da sociedade para a concretização da política pública. (KOERICH et al., 2009)

Anterior ao pacto em defesa do SUS, havia uma situação de fragilidade do processo de controle social. Os conselhos de saúde, no exercício da sua função de fiscalizadores, ficavam sobrecarregados com as inúmeras contas, rubricas e despesas constantes da gestão financeira, decorrente do repasse de recursos que era fragmentado por programa ou ação de saúde e condicionado à adesão dos municípios ao respectivo programa ou ação (MINISTÉRIO DA SAÚDE, 2006).

O pacto de gestão é a terceira dimensão do PS e aloca as diretrizes para a gestão do sistema nos aspectos de descentralização, regionalização, financiamento, planejamento, programação pactuada e integrada, regulação, participação social e gestão do trabalho e de educação em saúde (FADEL et al., 2009).

As formas de transferência dos recursos federais para estados e municípios foram alteradas pelo Pacto pela Saúde, passando a ser integradas em cinco grandes blocos de financiamento, Atenção Básica, Média e Alta Complexidade da Assistência, Vigilância em Saúde, Assistência Farmacêutica e Gestão do SUS, suprindo a anterior forma fragmentada de repasse (NÓBREGA et al., 2010).

Os municípios incluídos nos PS têm seus recursos repassados fundo a fundo, definido como modalidade preferencial de transferência de recursos. O uso dos recursos federais para o custeio fica restrito a cada bloco, atendendo às especificidades previstas nos mesmos, conforme regulamentação específica. As bases de cálculo que formam cada Bloco e os montantes financeiros destinados para os Estados, Municípios e Distrito Federal devem compor memórias de cálculo, para fins de histórico e monitoramento (BRASIL, 2006).

Em Minas Gerais, o número de municípios que aderiram ao termo de compromisso dos gestores (TCG) que discrimina as responsabilidades assumidas por cada município, especificadas em formato de um formulário e de um sistema de informação o SISPACTO, e os incluem no PS é de 828 municípios num total de 853 (CONASEMS, 2009). 


\section{3. EFICIÊNCIA NO SETOR DE SAÚDE}

Em um país de alta demanda pelos serviços públicos onde há escassez de recursos do setor, é de grande relevância a obtenção de maior eficiência nos investimentos governamentais. A avaliação eficaz desses programas pode contribuir para alcance de melhores resultados levando a um melhor emprego e controle dos recursos aplicados (COSTA; CASTANHAR, 2003).

Nessa direção, diversos trabalhos têm se dedicado a compreender a eficiência no setor de saúde como forma de exercer a accountability e garantir a qualidade dos investimentos públicos nesse setor, dentre eles destacam-se Pereira-Silva et. al. (2012); Brunozi Júnior et al (2011); Fonseca e Ferreira (2009); Galvarro et. al. (2012); Gonçalves et. al. (2012).

No setor de saúde são alocados os principais recursos públicos para cuidar do valor mais importante do ser humano: a vida. A análise da eficiência nesse setor é imprescindível para a formulação de políticas públicas de saúde, uma vez que, as políticas são formuladas com base nos dados obtidos, observando-se também as possibilidades dentro dos escassos recursos destinados a área. (MARINHO, 2003).

Pelo fato de os recursos públicos serem escassos e finitos, é importante determinar onde investir e o que universalizar, considerando a diferença entre os princípios do SUS e sua execução como política pública em um país com grandes desigualdades sociais e regionais, em que a pobreza e a exclusão social exigem políticas públicas adequadas, nesse sentido, a discussão sobre o processo de accountability e a eficiência na utilização dos recursos públicos ganham importância crescente, assim como a execução político-institucional das prefeituras e a competência da gestão local. (BODSTEIN, 2002)

Alcançar financiamento suficiente é imperativo, mas ter os recursos financeiros não assegura a cobertura universal, para que se possa garantir acesso à saúde a toda população é importante que os recursos sejam utilizados de maneira eficiente. (RELATÓRIO MUNDIAL DE SAÚDE, 2010)

\section{METODOLOGIA}

Este trabalho trata-se de uma pesquisa descritiva que, de acordo com Gil (2002), tem como objetivo principal a definição das características de uma dada população, fenômeno ou estabelece correlações entre variáveis. A pesquisa tem abordagem quantitativa, ou seja, analisa o que pode ser quantificável, sendo assim, transforma as informações em números para assim classificá-las e analisá-las utilizando-se de recursos e técnicas estatísticas (KAUARK; MANHÃES; MEDEIROS, 2010).

O procedimento técnico principal utilizado na coleta de dados foi a pesquisa documental, por meio de relatórios, documentos e estatísticas, acessados por meio eletrônico, em sites oficiais, sendo DATASUS e Ministério da Saúde que informam os indicadores e resultados de pesquisas na área da saúde. Os dados foram coletados de forma indireta, através do DATASUS e têm como fonte o Ministério da Saúde, através do Cadastro Nacional dos Estabelecimentos de Saúde no Brasil, Sistema de Informações sobre cobertura de atendimento do Programa de Agentes Comunitários de Saúde e de Saúde Bucal. Foram usadas as informações disponíveis em diversas tabelas da base de dados da pesquisa, utilizando, como referência, o período de Dezembro/2011. O desenvolvimento do presente trabalho contou também com pesquisa bibliográfica, por meio de livros, teses e artigos já publicados. 
A pesquisa contou com a participação de um conjunto ${ }^{2}$ de municípios de Minas Gerais, que compõem as diferentes microrregiões do estado, conforme divisão territorial do IBGE. Para a seleção foram combinadas duas técnicas amostrais: primeiro intencional em que foram selecionadas municípios que já aderiram ao Termo de Compromisso e Gestão, ou seja, que já estão incluídos no Pacto pela Saúde e que possuem população conforme os dados do IBGE, superior a 10.000 e inferior a 150.000 pessoas, para garantir a homogeneidade da amostra. Na sequência, foram escolhidos desses, 30 municípios, aleatoriamente. Em relação à unidade da federação escolhida, cabe mencionar que Minas Gerais foi selecionado por possuir um alto grau de adesão dos municípios ao Pacto pela Saúde, aumentando assim o número de municípios que se encaixavam dentro dos parâmetros da pesquisa.

Os dados foram coletados no intuito de medir a eficiência do setor de saúde, após o Pacto pela Saúde, em diferentes municípios, foram escolhidas 04 variáveis que representam os recursos, inputs, e outras 02 variáveis que representam os serviços ou produtos, outputs. Para a manipulação quantitativa dos dados foram usados os programas Microsoft Excel versão Office 2007®e StatisticalPackage for the Social Sciences - SPSS v. 15.0®.

As variáveis analisadas pertencem a dois programas do Ministério da Saúde sendo eles o Programa de Agente Comunitário de Saúde e o Programa de Saúde Bucal, que recebem seus recursos financeiros por meio de um bloco de financiamento do Pacto pela Saúde, e foram escolhidos por terem grande cobertura nos municípios mineiros.

Recursos/Insumos - inputs (X):

X1: Número de Agente comunitário de Saúde que atuam no Programa Saúde da Família.

X2: Valor em reais recebido pelo município destinado exclusivamente ao Programa Agente Comunitário de Saúde

Produtos/Serviços - outputs (X):

- Número de pessoas cobertas pelo Programa Agente Comunitário Saúde.

Recursos/Insumos - inputs (Y):

Y1: Número de auxiliares de saúde bucal e dentistas que atuam no Programa de Saúde Bucal.

Y2: Valor em reais recebido pelo município destinado exclusivamente ao Programa de Saúde Bucal.

Produtos/Serviços - outputs (Y):

- Número de pessoas cobertas pelo Programa de Saúde Bucal.

\subsection{MENSURAÇÃO DA EFICIÊNCIA}

Uma possibilidade para aferir eficiência de unidades produtivas é empregar fronteiras de produção, pois refletem a tecnologia usada, já que a estimativa de uma fronteira de produção é influenciada pelas unidades de melhor desempenho dentro da amostra de unidades analisadas. A função de produção representa as melhores práticas e, assim, as eficiências das unidades podem ser medidas (CASADO; SOUZA, 2007).

Neste trabalho, para o cálculo da eficiência, foi utilizada a Análise Envoltória de Dados (DEA), pois, de acordo com Lima e Bragança (2011), uma vez encontrada a fronteira eficiente, é possível identificar os benchmarks, ou seja, as DMUs que serão adotadas como

\footnotetext{
${ }^{2}$ A relação completa dos municípios encontra-se anexa ao trabalho.
} 
referência para aquelas ineficientes, dessa forma, os municípios que obtiverem os melhores resultados poderão servir de modelo aos que tiverem um baixo desempenho. A DEA e os dois modelos clássicos CCR e BCC, que foram utilizados nessa pesquisa, serão discutidos abaixo.

A Análise Envoltória de Dados (DEA) é um método não paramétrico para a determinação de fronteiras de produção. Essa fronteira de produção ou tecnologia é descrita como a máxima quantidade de outputs que pode ser conseguida, em relação aos inputs empregados em um processo de produção. A eficiência avaliada pela DEA é relativa, e usa por base observações reais, ou seja, as unidades tomadoras de decisão (DMUs) têm suas atuações aferidas por meio da comparação de seus resultados e dos seus insumos com os resultados e insumos das demais DMUs da amostra (FARIA; JANNUZZI; SILVA, 2008).

O conjunto de DMUs deve ser homogêneo e apresentar uso dos mesmos inputs e a produção dos mesmos outputs no qual a fronteira eficiente de produção será aquela que representa as unidades analisadas que conseguem maximizar a utilização dos inputs na produção de outputs, ou também consegue produzir uma quantidade maior de outputs com uma quantidade inferior de inputs. (CASADO e SOUZA, 2007)

Existem dois modelos DEA clássicos: o modelo CRS, chamado também de CCR (1) (Charnes, Cooper e Rhodes), que considera retornos de escala constantes, e o segundo modelo é o VRS, também conhecido por BCC (2) (Banker, Charnes e Cooper), que considera retornos variáveis de escala e não admite proporcionalidade entre inputs e outputs (MEZA et al.,2003).

De acordo com Mariano, Almeida e Rebelatto (2006), a avaliação da eficiência através da análise envoltória de dados pode ser sistematizado considerando-se os modelos, as hipóteses, o tipo de eficiência e a forma de fronteira, como pode ser melhor visualizado na tabela1.

Tabela 1- Resumo das propriedades dos modelos CCR e BCC.

\begin{tabular}{c|cccc}
\hline Técnica & Modelos & Hipóteses & Tipo de eficiência & Forma de fronteira \\
\hline $\begin{array}{c}\text { Análise por envoltória } \\
\text { de dados }\end{array}$ & CCR & $\begin{array}{c}\text { Retornos constantes } \\
\text { de escala }\end{array}$ & Eficiência total & Reta de $45^{\circ}$ \\
& BCC & $\begin{array}{c}\text { Retornos variáveis de } \\
\text { escala }\end{array}$ & Eficiência técnica & Linear por partes \\
\hline
\end{tabular}

Fonte: Mariano, Almeida e Rebelatto (2006)

A principal diferença entre os dois modelos está no levantamento sobre os retornos de escala de cada modelo. No modelo CCR, presumi-se que haja, entre insumos e produtos, retorno constante de escala enquanto no modelo BBC que exista retornos variáveis de escala. (OLIVEIRA, MACEDO e CORRAR, 2011)

O modelo CCR possibilita uma avaliação objetiva da eficiência como um todo e aponta as fontes e estimativas de montantes das ineficiências apontadas e o modelo BCC distingue entre ineficiências técnicas e de escala, calculando a eficiência técnica, a uma determinada escala de operações, e reconhecendo se estão presentes ganhos de escala decrescentes, crescentes e constantes, para futura análise. ( KASSAI, 2002)

\section{RESULTADOS}

A Tabela 2 apresenta as variáveis empregadas no modelo de eficiência, e suas estatísticas descritivas. Percebe-se, pelos dados, grande variabilidade entre os municípios, em 
especial em relação às variáveis de saída. Tal condição é normal, em decorrência, principalmente, das características dos municípios que variam entre 10 e 150 mil habitantes. Fatores esses que refletem sobre os insumos (recursos, pessoal) e sobre os produtos (recurso pessoal e pessoas cobertas). Já as assimetrias e curtoses são moderadas e não comprometem a análise que é, predominantemente, não paramétrica.

Tabela 2. Estatística descritiva das variáveis empregadas no modelo de eficiência dos municípios de MG

\begin{tabular}{ccccccccc}
\hline & N & Mínimo & Máximo & Média & $\begin{array}{c}\text { Desvio- } \\
\text { Padrão }\end{array}$ & Variância & Assimetria & Curtose \\
\hline $\begin{array}{c}\text { Recurso } \\
\text { R } \$ \text { X }\end{array}$ & 30 & $119.196,00$ & $1.882 .794,00$ & $939.862,27$ & $505.210,41$ & $255.237 .562 .360,34$ & - & - \\
$\begin{array}{c}\text { Pessoal } \\
\text { X }\end{array}$ & 30 & 15,00 & 178,00 & 94,43 & 47,57 & $2.262,94$ & 0,06 & 0,97 \\
$\begin{array}{c}\text { Pessoas } \\
\text { cobertas } \\
\quad \text { X }\end{array}$ & 30 & $11.858,00$ & $120.441,00$ & $51.182,43$ & $27.754,14$ & $770.292 .071,29$ & 0,56 & - \\
\hline \\
$\begin{array}{c}\text { Recurso } \\
\text { R Y }\end{array}$ & 30 & $27.300,00$ & $645.300,00$ & $206.939,50$ & $160.431,00$ & $25.738 .106 .235,09$ & 1,03 & 0,11 \\
$\begin{array}{c}\text { Pessoal } \\
\text { Y }\end{array}$ & 30 & 4,00 & 58,00 & 18,77 & 12,80 & 163,84 & 1,24 & 1,54 \\
$\begin{array}{c}\text { Pessoas } \\
\text { Cobertas } \\
\quad \text { Y }\end{array}$ & 30 & $3.453,00$ & $96.603,00$ & $29.993,57$ & $21.724,48$ & $471.952 .819,70$ & 1,24 & 1,59 \\
\hline
\end{tabular}

Fonte: Resultados da pesquisa.

Pela distribuição dos escores de eficiência técnica, exibida no tabela 3, é possível observar o nível de eficiência dos municípios, permitindo que os agentes públicos criem políticas que visem a uma melhor prestação dos serviços e a otimização dos recursos no setor de saúde.

Os resultados do modelo com retornos constantes e com retornos variáveis são apresentados de forma comparativa, uma vez que, não se objetivou aplicar a postura conservadora ou, tão pouco, parcimoniosa na escolha da apresentação. Logo, para efeito de benchmark o modelo CCR é mais indicado e para a introdução de políticas de ação ou de desempenho o modelo BCC é mais inclusivo.

Tabela 3 . Escores de eficiência dos municípios de MG

\begin{tabular}{lcc}
\hline & $\begin{array}{c}\text { Modelo RCC (Retornos } \\
\text { Constantes) }\end{array}$ & Modelo BCC (Variáveis) \\
\hline Alfenas & $100,00 \%$ & $100,00 \%$ \\
Araxá & $80,84 \%$ & $81,00 \%$ \\
Barbacena & $86,24 \%$ & $96,61 \%$ \\
Bocaiuva & $81,02 \%$ & $86,64 \%$ \\
Bom Despacho & $100,00 \%$ & $100,00 \%$ \\
Caratinga & $76,37 \%$ & $82,33 \%$ \\
Conceição do Mato Dentro & $100,00 \%$ & $100,00 \%$ \\
Conselheiro Lafaiete & $100,00 \%$ & $100,00 \%$ \\
Curvelo & $91,32 \%$ & $91,97 \%$ \\
Diamantina & $82,94 \%$ & $83,68 \%$ \\
Esmeraldas & $100,00 \%$ & $100,00 \%$ \\
Espera Feliz & $61,94 \%$ & $66,37 \%$ \\
Formiga & $93,17 \%$ & $100,00 \%$ \\
Frutal & $84,84 \%$ & $84,96 \%$ \\
Grão Mogol & $79,60 \%$ & $81,11 \%$ \\
Itabira & $84,68 \%$ & $99,84 \%$ \\
Itaguara & $99,32 \%$ & $100,00 \%$ \\
\hline
\end{tabular}




\begin{tabular}{lcc}
\hline Janauba & $82,95 \%$ & $94,67 \%$ \\
Lavras & $97,25 \%$ & $99,13 \%$ \\
Lima Duarte & $94,38 \%$ & $97,09 \%$ \\
Manhuaçu & $100,00 \%$ & $100,00 \%$ \\
Muriáe & $100,00 \%$ & $100,00 \%$ \\
Nova Serrana & $82,62 \%$ & $85,14 \%$ \\
Ouro Preto & $100,00 \%$ & $100,00 \%$ \\
Pará de Minas & $90,79 \%$ & $100,00 \%$ \\
Patos de Minas & $100,00 \%$ & $100,00 \%$ \\
Ponte Nova & $91,07 \%$ & $93,73 \%$ \\
Ubá & $100,00 \%$ & $100,00 \%$ \\
Viçosa & $98,17 \%$ & $98,73 \%$ \\
Visconde de Rio Branco & $88,00 \%$ & $90,49 \%$ \\
\hline
\end{tabular}

Fonte: Resultados da pesquisa.

Embora a média da eficiência seja elevada nos dois modelos, a Tabela 3, destaca-se uma grande amplitude média de variação dos escores de eficiência que distribuem-se de 62 a 100 (modelo RCC) e 66 a 100 (modelo BCC). Portanto, faz-se necessário uma investigação mais apropriada das condições de distribuição e concentração dos dados.

Tal perspectiva de análise se encontra materializada na Tabela 4, em que são apresentadas as estatísticas descritivas para os escores de eficiência técnica dos municípios deMinas Gerais.

Tabela 4. Análise descritiva dos escores de eficiência técnica dos municípios de MG

\begin{tabular}{ccccccccc}
\hline & N & Mínimo & Máximo & Média & Desvio & Variância & Assimetria & Curtose \\
\hline $\begin{array}{c}\text { Modelo } \\
\text { RCC }\end{array}$ & 30 & $62 \%$ & $100 \%$ & $90,92 \%$ & $9,625 \%$ & 92,644 & $-1,005$ & 1,013 \\
$\begin{array}{c}\text { Modelo } \\
\text { BCC }\end{array}$ & 30 & $66 \%$ & $100 \%$ & $93,78 \%$ & $8,587 \%$ & 73,735 & $-1,477$ & 1,980 \\
\hline
\end{tabular}

Fonte: Resultados da pesquisa.

O coeficiente de curtose evidencia o achatamento da distribuição que, na categoria de platicúrtica, possui uma inclinação menor no distanciamento em torno da média, a assimetria negativa representa o impacto da influência de escores mais baixos nos níveis de eficiência.

A ocorrência de escores menores denota uma sutil calda à esquerda, que toca a marca mínima de $61,94 \%$ pelo modelo CCR e a de $66,37 \%$ pelo modelo BCC, a análise de tais dados conduz a observação de que mesmo no município no qual foi observado o grau de eficiência mais baixo o nível de aproveitamento ainda é aceitável de acordo com os critérios adotados, todavia, o objetivo do modelo de gestão em estudo é o de minimizar os prejuízos decorrentes das distorções observadas na alocação dos recursos, sendo de tal forma, o patamar tido como aceitável deve estar sujeito a contínuas ações de aprimoramento, uma vez que os números observados em outros municípios analisados chegam a escores de $100 \%$, o que demonstra que é possível atingir um nível de eficiência considerado ótimo pelos parâmetros da pesquisa.

As figuras 2 e 3 procuram ilustrar com mais propriedade essas observações.

Figura 2. Distribuição dos escores de eficiência da saúde em Minas Gerais pelo modelo BCC. 


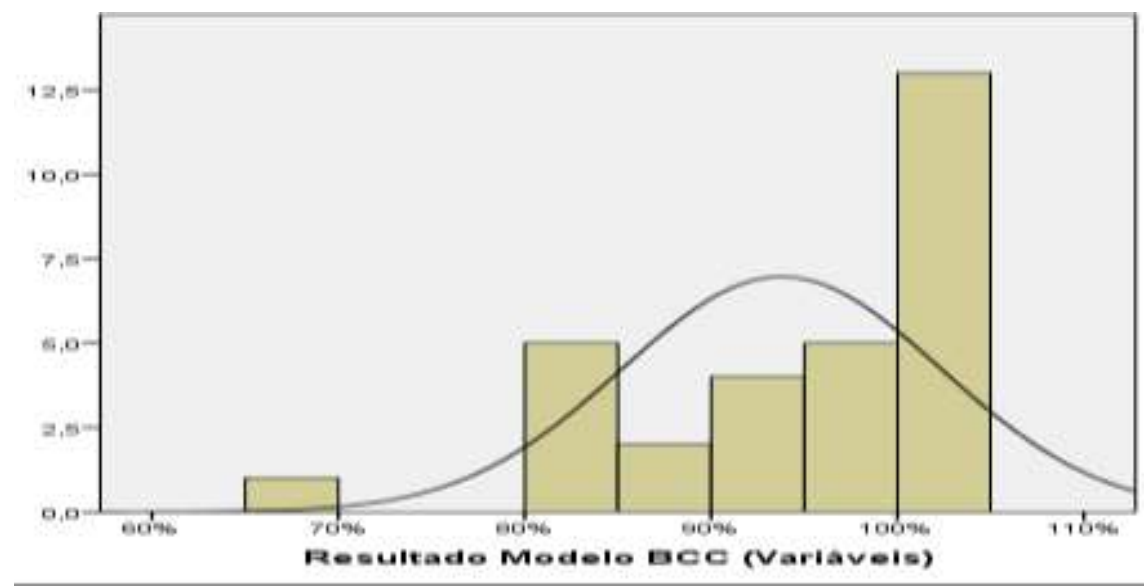

Fonte: Resultados da pesquisa

Figura 3. Distribuição dos escores de eficiência da saúde em Minas Gerais pelo modelo CCR.

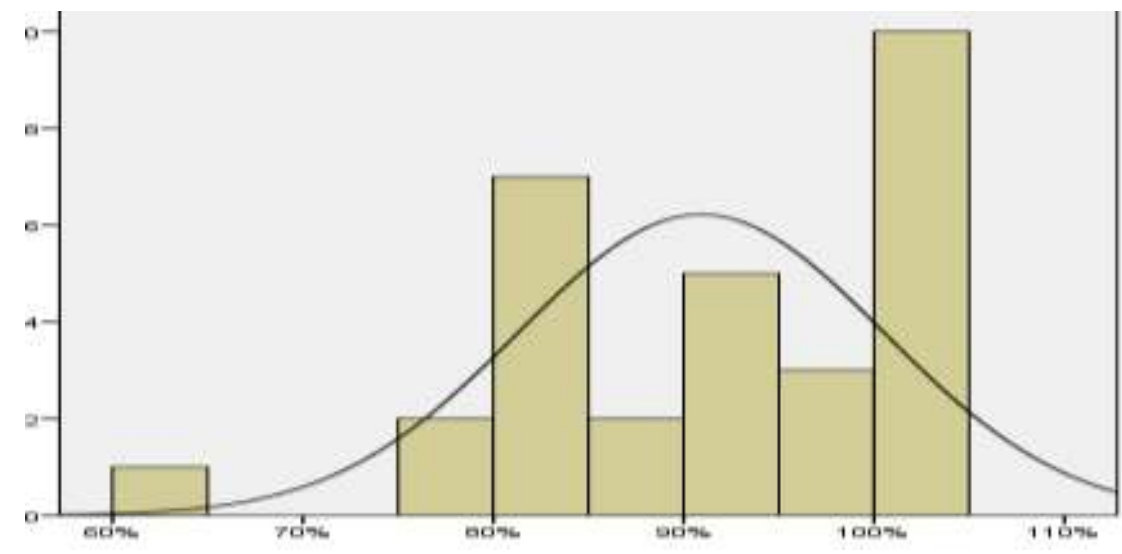

Fonte: Resultados da pesquisa

Para a avaliação qualitativa dos resultados foi construído um critério de categorização das DMUs em razão dos resultados, tomando-se como referência a média e o desvio-padrão, enquanto principais estatísticas descritivas apuradas. Desse modo, as organizações foram assim classificadas:

Tabela 5. Classificação de desempenho dos municípios, conforme escore de eficiência da abordagem DEA retornos constantes.

\begin{tabular}{ccc}
\hline Critério & Escores & $\begin{array}{c}\text { Desempenho na otimização dos } \\
\text { recursos }\end{array}$ \\
\hline $\begin{array}{c}\text { Inferior a Média menos 1 Desvio- } \\
\text { Padrão }\end{array}$ & $\mathrm{E}<86,11$ & Fraco \\
Média mais ou menos 1 Desvio- & Bom \\
$\begin{array}{c}\text { Padrão } \\
\text { Inferior a Média menos 1 Desvio- } \\
\text { Padrão }\end{array}$ & $86,11<\mathrm{E}<95,73$ & Excelente \\
\hline
\end{tabular}

Fonte: Resultado da pesquisa

De acordo com os resultados apresentados, a média de eficiência dos municípios pelo modelo foi de CCR 90,92\% e o desvio padrão de 9,625\%, pelo modelo BCC 93,78\% e desvio padrão de $8,587 \%$. 
Esses resultados demonstram que 13 municípios que representam 43,33\% da amostra pelo modelo CCR, apresentam escores excelentes de desempenho. Desses, 10 municípios apresentaram escores máximos, representando, portanto, benchmarks para políticas públicas no setor, tendo em vista, principalmente, sua capacidade relativa de aproveitamento de recursos humanos e materiais.

Pelo modelo BCC, 18 municípios, que representam 60\% da amostra, apresentaram escores de eficiência excelentes dos municípios, desses, 13 apresentam escores máximos de eficiência.

Com escores médios de eficiência, encontra-se ou 7 municípios ou 23,33\% pelo modelo CCR e 16,66\% ou 5 municípios pelo modelo BCC.

O restante da amostra, 10 municípios, representando 33,33\% da amostra, apresentaram escores de baixo desempenho pelo modelo CCR. Pelo modelo BCC 7 ou 23,33\% apresentaram escores de fraco desempenho.

Em um trabalho sobre a eficiência nos setor de saúde Fonseca e Ferreira (2009) analisaram unidades institucionais homogêneas em municípios do estado de Minas Gerais, no qual se constatou disparidades na gestão dos recursos da saúde, assim como foram encontradas no presente estudo, o que evidencia a necessidade das trocas de experiências, entre os gestores municipais, objetivando diminuir tais diferenças. ${ }^{3}$

Entretanto, como observado no presente trabalho, dois terços dos municípios analisados obtiveram desempenho considerado bom, ou seja, nos quesitos de eficiência e desempenho, o Pacto pela Saúde tem atingido seus resultados, o que corrobora as observações de Fadel et al. (2009); Guerreio e Branco, (2011);Koerichet al. (2009); Pereira et al. (2009); no que se refere aos aspectos positivos do Pacto já discorridos neste trabalho.

\section{CONSIDERAÇÕES FINAIS}

Os resultados evidenciaram que o desempenho dos municípios no que se refere à otimização de recursos pode ser considerado bom, uma vez que, a maior parte deles concentrarem-se em escores superiores à média, todavia se faz necessário um contínuo aprimoramento neste processo, para que se alcance em todos eles o mesmo nível de excelência que foi encontrado em parte da amostra.

Ao se tomar os municípios com desempenho relativo máximo, contrapostos pelo grupo de municípios com desempenho relativo reduzido, pode-se observar uma margem percentual de possível melhora que chega a ser de até 38,06 \% pelo modelo CCR e de 33,63 $\%$ pelo modelo BCC.

Ao se analisar os municípios que apresentaram escores de fraco desempenho, observase que, ainda, há um grande potencial relativo de aprimoramento, potencial este que se explorado resultaria em significativas melhoras para a população assistida.

Os resultados alcançados por este estudo permitem concluir que o modelo de gestão proposto pelo Pacto pela Saúde funciona nos setores analisados e que estes tiveram como desempenho mínimo um bom resultado. Pode se concluir ainda que, nos municípios onde foram apurados índices menores, se faz necessário um acompanhamento, que busque identificar quais fatores interferiram negativamente nos resultados obtidos, para assim assegurar uma crescente melhoria dos serviços prestados.

Nos resultados podem ser identificadas cidades que servem de benchmark para as demais, o que corrobora a premissa de que um constante diálogo entre os gestores

\footnotetext{
${ }^{3}$ Embora seja oportuno ressaltar que por ser a DEA uma abordagem de fronteira de eficiência relativa, comparações diretas só podem ser realizadas em DMUs pertencentes à mesma fronteira.
} 
Revista de Administração Hospitalar, v.11, n.1, pp. 83-98, janeiro/março, 2014/ Kerla Fabiana Dias Cabral e Marco Aurélio Marques Ferreira

intermunicipais, com troca de experiências, poderia ser válido como estratégia para otimizar as práticas de gestão.

\section{REFERÊNCIAS BIBLIOGRÁFICAS}

ALBUQUERQUE, P. H. Os impactos econômicos da CPMF: teoria e evidências. Brasília: Banco Central do Brasil, jun. 2001 (Trabalhos para Discussão, 21).

BRASIL. Constituição Federal, 5 de outubro de 1988.

Ministério da Saúde. CONASEMS. Reflexões aos Novos Gestores Municipais de Saúde. Brasília: CONASEMS; 2009. Disponível em: http://www.conasems.org.br/files/reflexoes_novos_gestores.pdf Acesso em: 25 de abril 2012.

.Ministério da Saúde. Dialogando sobre o Pacto pela saúde. Brasília: 2006. Textos Básicos de Saúde. Disponível em: http://portal.saude.gov.br/portal/arquivos/pdf/dialogando_sobre_pacto_pela_saude.pdf.

Acesso em: 24 de abril 2012.

Ministério da Saúde. Consulta de pagamentos. Disponível em: http://www.fns.saude.gov.br/Acesso em: 13 de agosto de 2012.

BARROSO, R. F. F. A Universidade e as políticas públicas de saúde. Revista Texto e Contexto Enfermagem. v.16, n.3 Florianópolis July/Sept. 2007.

BODSTEIN, R. Atenção Básica na Agenda da Saúde. Ciência e Saúde Coletiva. Rio de Janeiro. v. 7, 2002.

BRUNOZI JÚNIOR, A. C.; ABRANTES, L. A.; FERREIRA, M. A.. M.; KLEIN, T. C. Aplicação do modelo de regressão logística para análise dos efeitos das transferências intergovernamentais e arrecadação tributária sobre os indicadores sociais da saúde e educação em Minas Gerais. Revista de informação contábil (UFPE), 2011.

CAMPELli, M. G. R; CALVO, M. C. M. O cumprimento da Emenda Constitucional $n^{o} .29$ no Brasil. Cadernos Saúde Pública,v.23, n.7,Rio de Janeiro, July, 2007..

CARVALHO, E. C. Finanças Públicas e Estabilização no Plano Real: Uma Reinterpretação. PUCSP, abr. 2001. Textos para discussão.

CARVALHO, G. Financiamento da saúde pública no Brasil no pós- constitucional de 88. Actas de Saúde Coletiva, v.2, n.2, p.39-51, 2008.

CARVALHO, G. A inconstitucional administração pós-constitucional do SUS através de normas operacionais. Revista Ciência \& Saúde Coletiva.v. 6, n. 2, p. 435-444, 2001.

CASADO, F. L.; SOUZA, A. M. Análise Envoltória de Dados: Conceitos, Metodologia e Estudo da Arte na Educação Superior. Revista do Centro de Ciências Sociais e Humanas, Universidade Federal de Santa Maria, v. 1, p. 1-154, 2007.

COSTA, F. L.; CASTANHAR, J. C. Avaliação de programas públicos: desafios conceituais e metodológicos. Revista de Administração Pública, FGV.v. 37, n. 5, p. 969-92, set./out. 2003.

COUTTOLENC, B. F.; ZUCCHI, P. Gestão de Recursos Financeiros: Para gestores municipais de serviços de saúde. USP Série Saúde \& Cidadania, v. 10: São Paulo, 1998.

DATASUS. Informações de saúde. Disponível em: http://cnes.datasus.gov.br/Index.asp?home=1Acesso em 5 de Agosto de 2012. 
Revista de Administração Hospitalar, v.11, n.1, pp. 83-98, janeiro/março, 2014/ Kerla Fabiana Dias Cabral e Marco Aurélio Marques Ferreira

FADEL, C. B. et al. Administração Pública: o pacto pela saúde como uma nova estratégia de racionalização das ações de saúde no Brasil. Revista de Saúde Pública,Rio de Janeiro, v. 43, n 2, p. 445-456, 2009.

FARIA,F. P; JANNUZZI, P. M.; SILVA, J. S. Eficiência dos gastos municipais em saúde e educação: uma investigação através da análise envoltória no estado do Rio de Janeiro. Revista da Administração Pública, Rio de Janeiro,v. 42, n.1, p. 155-177, jan/fev, 2008.

FONSECA, P. C.; FERREIRA, M. A. M. Investigação dos níveis de eficiência na utilização de recursos no setor de saúde: uma análise das microrregiões de Minas Gerais. Revista Saúde e Sociedade, v. 18, n. 2, p. 199-213, 2009.

FONTOURA, R.. T. MAYER, C. N. Uma breve reflexão sobre a integralidade. Revista Brasileira Enfermagem, v. 59, n. 4, p. 532-7. jul-ago,2006.

FRUTUOSO, J. A gestão do sistema único de saúde. Brasília. UNESCO, p. 89-105, 2010.

GALVARRO, M. P. S. Q. S.; FARIA. E. R.; FERREIRA, M. A. M. Desigualdades regionais na saúde no Estado de Minas Gerais. RAHIS. Revista de Administração Hospitalar e Inovação em Saúde, v. 8, n. 8, p.12-27, 2012.

GARRAFA, V. Saúde pública, bioética e equidade. Revista Bioética, v.5 n.1 p.27-33. 1997.

GIL, A. C. Como Elaborar Projetos de Pesquisa. 4. ed. - São Paulo: Atlas, 2002.

GUERREIRO, J. V.; BRANCO, M. A. F. Dos pactos políticos à política dos pactos na saúde. Revista Ciência\& Saúde Coletiva, v.16, n. 3, p.1689-1698, 2011.

GONÇALVEZ, M. A.; SANTOS, L. M.; FERREIRA, M. A. M. Uma Análise da Mudança de Produtividade da Alocação de Recursos Públicos na Atenção Básica da Saúde em Municípios da Região Sudeste Brasileira. Revista de Ciências da Administração, v. 14, n. 34 (CAD/UFSC), Florianópolis, 2012.

KASSAI, S. Utilização da Análise Envoltória de Dados (DEA) na Análise de Demonstrações Contábeis. Tese de doutorado - Faculdade de Economia, Administração e Contabilidade da Universidade de São Paulo, 2002.

KAUARK,F. S. MANHÃES, F. C. e MEDEIROS, C. H. Metodologia da pesquisa:um guia prático. Itabuna: Via Litterarum, 2010.

KOERICH, M. S. et al. Pacto em defesa da saúde:divulgando os direitos dos usuários pela pesquisa-ação. Revista Gaúcha Enfermagem, Porto Alegre (RS), v.30, n.4, p. 677-84. 2009.

LUCCHESE, P. T. R. Descentralização do Financiamento e Gestão da Assistência à Saúde no Brasil: A Implementação do Sistema Único de Saúde-Retrospectiva 1990/1995. Revista Planejamento e Políticas Públicas/ IPEA, v. 14, p. 75-156, 1996.

LEVCOVITZ, E.; LIMA, L. D.; MACHADO, C. V. Política de saúde nos anos 90relações intergovernamentais e o papel das Normas Operacionais Básicas. Revista Ciência \& Saúde Coletiva, v.6, n.2, p.269-291, 2001.

LEVI, M. L. SCATENA, J. H. G. Avaliação nacional das comissões inter gestores bipartites (cibs): as cibs e os modelos de indução da regionalização no SUS: Evolução recente do financiamento do SUS e considerações sobre o processo de regionalização. 2010. Disponível em: <http://bvsms.saude.gov.br/bvs/descentralizacao/cibs/pdf/RelatorioFinanciamento_outubr o_2010.pdf>Acesso em 5 de agosto de 2012. 
MACHADO, R. R.et al. Entendendo o pacto pela saúde na gestão do SUS e refletindo sua implementação. Revista Eletrônica de Enfermagem. 2009. Disponível em: <http://www.fen.ufg.br/revista/v11/n1/pdf/v11n1a23.pdf> Acesso em: 28 de abril 2012.

MARQUES, R. M. O Financiamento do Sistema Público de Saúde Brasileiro. Comissão Econômica para América Latina e Caribe. Santiago de Chile, 1999. Disponível em <http://www.eclac.cl/publicaciones/xml/7/4507/lcl1233p.pdf>. Acesso em:29 abril 2012.

MARQUES, R. M. MENDES, A. Os dilemas do financiamento do SUS no interior da seguridade social. Revista Economia e Sociedade, Campinas, v. 14, n. 1 (24), p. 159-175, jan./jun. 2005.

MARINHO, A. Avaliação da eficiência técnica nos serviços de saúde nos municípios do Estado do Rio de Janeiro. Revista Brasileira de Economia, v.57, n.3. Rio de Janeiro July/Sep. 2003.

MEDICE, A. Breves Considerações sobre a Relação entre Financiamento da Saúde e Direito Sanitário no Brasil In: BRASIL - Ministério da Saúde, 2009.

MEZA, L. A. SIAD - Sistema integrado de apoio à decisão: uma implementação computacional de modelos de análise de envoltória de dados. In: Simpósio de pesquisa operacional da marinha, 6., 2003, Rio de Janeiro. Anais... CASNAV, 2003. Disponível em: <http://www.cnpm.embrapa.br/publica/download/newsdownload/artigos_resumos\%20anais\% 20eventos/apc_6spolm03_siad_eg.pdf> Acesso em: 15 de agosto de 2012.

MODESTO, P. Notas para um debate sobre o princípio da eficiência. Revista do Serviço Público, n. 2, p. 105-119, 2000.

MARIANO, E. B.; ALMEIDA, M. R.; REBELATTO, D. A. N. Peculiaridades da Análise por Envoltória de Dados. Anais do XII SIMPEP, 2006.

NÓBREGA, C. B. C. et.al. Financiamento do setor saúde: uma retrospectiva recente com uma abordagem para a odontologia. Revista Ciência\& Saúde Coletiva, p. 1763-1772, 2010.

OLIVEIRA, V. H.; MACEDO, M. A. S.; CORRAR, L. J. Estudo do desempenho dos maiores bancos de varejo no Brasil por meio da análise envoltória de dados (DEA). Revista de Informação Contábil ,v. 5, no 2, p. 01-20, Abr-Jun/2011.

PAIM, J. et al. The Brazilian health system: history, advances and challenges. Lancet, 2011.

PASCHE, D. F. et al. Paradoxos das políticas de descentralização de saúde no Brasil. RevistaPanamericanaSaludPublica/Pan Am J Public Health, v.20, n.6, 2006.

PEIXOTO, S .D. G. et .al. Decentralization and corruption: evidence from primary healthcare programs. Applied EconomicsLetters (Print), v. 19, p. 1885/18-1888, 2012.

PEREIRA, C. Discricionariedade, eficiência e controle dos atos administrativos. $2^{\circ}$ Concurso de Monografias da Controladoria Geral da União. Belo Horizonte, 2007.

PEREIRA-SILVA, A. A.; FERREIRA, M. A. M.; BRAGA, M. J. ; ABRANTES, L. A. Eficiência na Alocação de Recursos Públicos Destinados à Educação, Saúde e Habitação em Minas Gerais. Revista Contabilidade, Gestão e Governança, v. 15, p. 96-114, 2012.

PEREIRA, M. J. B et al. A enfermagem no Brasil no contexto da força de trabalho em saúde: perfil e legislação. Revista Brasileira Enfermagem, Brasília; v. 62, n.5, p. 771-7.2009.

PRUD'HOMME,R. The dangers of decentralization.The World Bank Research Observer, 10: 201-222, 1995. 
RELATÓRIO MUNDIAL DE SAÚDE. Financiamento dos sistemas de saúde: O caminho para a cobertura universal. 2010.

SARAIVA, E. Introdução á teoria da política pública. Políticas públicas; coletânea, Brasília; V. 2. 2006.

SOUZA, R. R. A Regionalização no Contexto Atual das Políticas de Saúde. Revista Ciência e Saúde Coletiva, v. 6, n. 2, p. 451-455, 2001.

SOUZA, C. Políticas Públicas: uma revisão da literatura. Sociologias. Porto Alegre, ano 8, n 16 , p. 20-45,jul/dez 2006.

ANTI, E. M. D. ; PISCITELLI. T. S.; MASCITTO. A. Tributação, Responsabilidade Fiscal e Desenvolvimento. Caderno de Direito GV. Rio de Janeiro, v. 5, n 1, 2008. 